\title{
Indium Titanium Oxide Nanoparticles Induced Hepatic Damage: Hepatoprotective Role of Novel 2-Imino-4-methyl-1, 2-Dihydropyrimido [5, 4C] Quinoline-5(6H)-one
}

\author{
Dinesh Bheeman, ${ }^{1}$ Sinjula Cheerothsahajan, ${ }^{1}$ Sathish Sugumaran, ${ }^{2}$ \\ Sankaran Mathan, ${ }^{3}$ Ramesh Mathan, ${ }^{4}$ Sivanesan Dakshanamurthy, ${ }^{5}$ \\ Ranjithkumar Rajamani, ${ }^{6}$ and Chandar Shekar Bellan ${ }^{7}$ \\ ${ }^{1}$ Department of Biotechnology, Sree Narayana Guru College, Coimbatore, Tamil Nadu 641105, India \\ ${ }^{2}$ Nanotechnology Research Lab, Kongunadu Arts and Science College, Coimbatore, Tamil Nadu 641029, India \\ ${ }^{3}$ Department of Chemistry, Bharathiar University, Coimbatore, Tamil Nadu 641046, India \\ ${ }^{4}$ Unit of Toxicology, Department of Zoology, School of Life Sciences, Bharathiar University, Coimbatore, Tamil Nadu 641046, India \\ ${ }^{5}$ Lombardi Comprehensive Cancer Center, Georgetown University Medical Center, Washington, DC 20057, USA \\ ${ }^{6}$ Department of Biotechnology, Dr.N.G.P. Arts and Science College, Coimbatore, Tamil Nadu 641048, India \\ ${ }^{7}$ Nanotechnology Research Laboratory, Department of Physics, Kongunadu Arts and Science College, G N Mills Post, \\ Coimbatore, Tamil Nadu 641029, India
}

Correspondence should be addressed to Chandar Shekar Bellan; chandar.bellan@gmail.com

Received 2 August 2014; Revised 18 November 2014; Accepted 19 November 2014; Published 9 December 2014

Academic Editor: Kanji Yamasaki

Copyright ( 2014 Dinesh Bheeman et al. This is an open access article distributed under the Creative Commons Attribution License, which permits unrestricted use, distribution, and reproduction in any medium, provided the original work is properly cited.

\begin{abstract}
Protective role of 2-imino-4-methyl-1, 2-dihydropyrimido [5, 4C] quinoline-5(6H)-one (IMDHPQ) in indium titanium oxide nanoparticles (InTiO NPs) induced hepatotoxicity was analyzed. InTiO NPs were synthesized and given orally to albino rats to assess their hepatotoxicity. NPs mediated oxidative stress and liver tissue pathology were analyzed. Altered antioxidants (GSH, GPx, and catalase) and, biochemical (SGOT, SGPT, ALP, total protein, and total bilirubin) and histopathological changes were observed due to the oxidative stress caused by InTiO NPs. Varying effects of IMDHPQ on each parameter were observed in the present study. The altered parameters of InTiO NPs exposed rats might be due to the oxidative stress caused by NPs and hepatoprotective or ameliorative efficacy of quinoline compound IMDHPQ on signaling and molecular mechanism needs further study.
\end{abstract}

\section{Introduction}

With the new advent of nanotechnology, industries are extensively exploiting the methods involved in nanotechnology $[1,2]$. Constant exposure to the nanoparticles will induce toxic effects. It may cause many other acute and chronic toxic effects like damage to kidney, liver, and lungs and some nanoparticles are carcinogenic [3]. Nanoparticles can translocate through circulatory, lymphatic, and nervous systems to various tissues and organs and even to brain [4]. Due to the minute size, nanoparticles can penetrate through basic biological structures, impairing their normal functions. Diseases associated with inhaled nanoparticles are asthma, lung cancer, bronchitis, emphysema, and neurodegenerative disorders. Nanoparticles may cause colon cancer, blood cancer, heart disease, and cardiac arrest. Translocation to various organs like liver, kidney, and spleen may damage the organ [5].

Titanium dioxide will synthesize free radicals which may cause oxidative damage to the DNA, but new nanomaterials may have both useful and toxic effects $[4,6]$. Indium compounds are highly carcinogenic to human. It also showed 
increased rates of hepatocellular neoplasm in mice when exposed to low dose for short period [7]. But the synergistic effect of indium and titanium oxide is yet to be known.

Liver is the largest organ in the human body which is situated in upper abdomen. It is involved in major metabolisms and excretion. The major functions of liver are carbohydrate, lipid, and protein metabolism, detoxification, storage of vitamins, and secretion of bile [8]. Hepatitis, cirrhosis, and alcohol-liver diseases are the severe liver diseases. Liver diseases are mainly caused by toxic chemicals, excess consumption of alcohol, infections, and autoimmune disorders. Most of the hepatotoxic chemicals damage liver cells by inducing lipid peroxidation and other types of oxidative damage [9]. Pyrimidoquinoline is the clubbed compound of both quinoline and pyrimidine. Pyrimidine has played a vital role in the manufacture of various biologically active drugs as antimicrobial, calcium channel blockers, antitubercular, antibacterial, and anti-inflammatory [10]. Many classes of chemotherapeutic agents containing pyrimidine nucleus are in clinical use. According to research, if pyrimidine and quinoline moieties are clubbed into one molecule, the resultant molecule may enhance the pharmaceutical activity [10].

Novel quinoline derivative synthesized by cyclisation is 2-imino-4-methyl-1, 2-dihydropyrimido [5, 4C] quinoline$5(6 \mathrm{H})$-one (IMDHPQ) and it is derived from the compound 4-hydroxy-3-acyl quinoline-2-one. Pyrimidoquinoline ring system has structural similarity to naturally occurring flavines. DPPH, FRAP, radical scavenging assays of this compound shown to have antioxidant effect [11]. Hence, the present study was aimed at investigating the hepatoprotective or ameliorative role of the quinoline compound IMDHPQ against InTiO NPs induced hepatotoxicity in experimental albino rats.

\section{Materials and Method}

2.1. Animals. Male albino rats weighing 100-150 g were procured from Sri Venkateshwara Enterprises, Bangalore, and acclimatized for one week under standard $12 \mathrm{~h}$ day/night cycle. They were fed with standard rat feed and water. Ethical clearance for experimental protocol and handling the animals was obtained from the Institutional Animals Ethical Committee prior to the beginning of the scientific investigation.

2.2. Drugs and Chemicals. The chemical compound IMDHPQ was a kind gift (Dr. Mathan S., University of Florida, USA). Standard drug silymarin was purchased commercially from Palakkad, India. SGOT, SGPT, ALP, bilirubin, and total protein kits were procured from Span Diagnostics, Surat, India. And the rest of the chemicals utilized were of analytical grade and were obtained from local source.

2.3. Preparation of Suspensions. Standard drug silymarin and InTiO NPs $(50 \mathrm{mg}$ ) were suspended in distilled water separately and used for in vivo investigations. The dosage of InTiO NPs was selected based on our previous report [12].
The nanoparticles were sonicated well before administration because they are known to form aggregates. Quinoline derivative IMDHPQ compound was dissolved in dimethyl sulfoxide to prepare the suspension. All drugs and inducers were administered orally to rats.

\subsection{Experimental Design}

Group 1: control (DMSO only) for 7 days.

Group 2: induced (received InTiO NPs $50 \mathrm{mg} / \mathrm{kg}$ of body weight) for 7 days.

Group 3: standard silymarin $(100 \mathrm{mg} / \mathrm{kg})$ InTiO NPs $(50 \mathrm{mg} / \mathrm{kg})$ for 7 days.

Group 4: pyrimidoquinoline $(500 \mu \mathrm{L} / \mathrm{kg})+\mathrm{InTiO}$ NPs $(50 \mathrm{mg} / \mathrm{kg})$ for 7 days.

Group 5: pyrimidoquinoline $(1000 \mu \mathrm{L} / \mathrm{kg})+$ InTiO NPs $(50 \mathrm{mg} / \mathrm{kg})$ for 7 days.

The animals were treated with respective treatments for 7 days and blood samples were collected under mild ether anesthesia; later the animals were sacrificed and liver tissues were harvested. All experiments were done in triplicate.

2.5. Assessment of Hepatotoxicity. The blood samples were drawn from all the animals by puncturing retroorbital plexus on 7 th day of the treatment. The blood samples were centrifuged immediately to get clear serum and subjected for estimation of various biochemical parameters, namely, SGOT (serum glutamate oxaloacetate transaminase), SGPT (serum glutamate pyruvate transaminase), ALP (alkaline phosphatase), serum total bilirubin, and total protein, according to the manufacturer's protocol (Span Diagnostic, Surat).

2.6. Assessment of Oxidative Stress. Liver tissue homogenate was used for the antioxidants like catalase [13] and phase II metabolizing enzymes GSH [14] and GPx [15] in experimental rats. Thiobarbituric acid reactive substances (TBARS) contents were estimated according to [16].

2.7. Histopathological Analysis. A portion of liver was carefully dissected out, washed with $0.9 \%$ normal saline solution, and preserved in $10 \%$ formaldehyde for histopathological observations. Thin sections were prepared and stained with hematoxylin and eosin dye for microscopic observations. The microscopic slides of the liver cells were photographed at a magnification of $40 x$.

2.8. Statistical Analysis. The data were analyzed by one way ANOVA using Graph Pad Prism Software. The comparison between groups was done by calculating the percentage reduction in each parameter compared to inducer group. Values are the mean \pm S.E.M. of six rats per treatment. Significance is marked as ${ }^{*} P<0.05,{ }^{* *} P<0.01$, and ${ }^{* * *} P<$ 0.001 . 

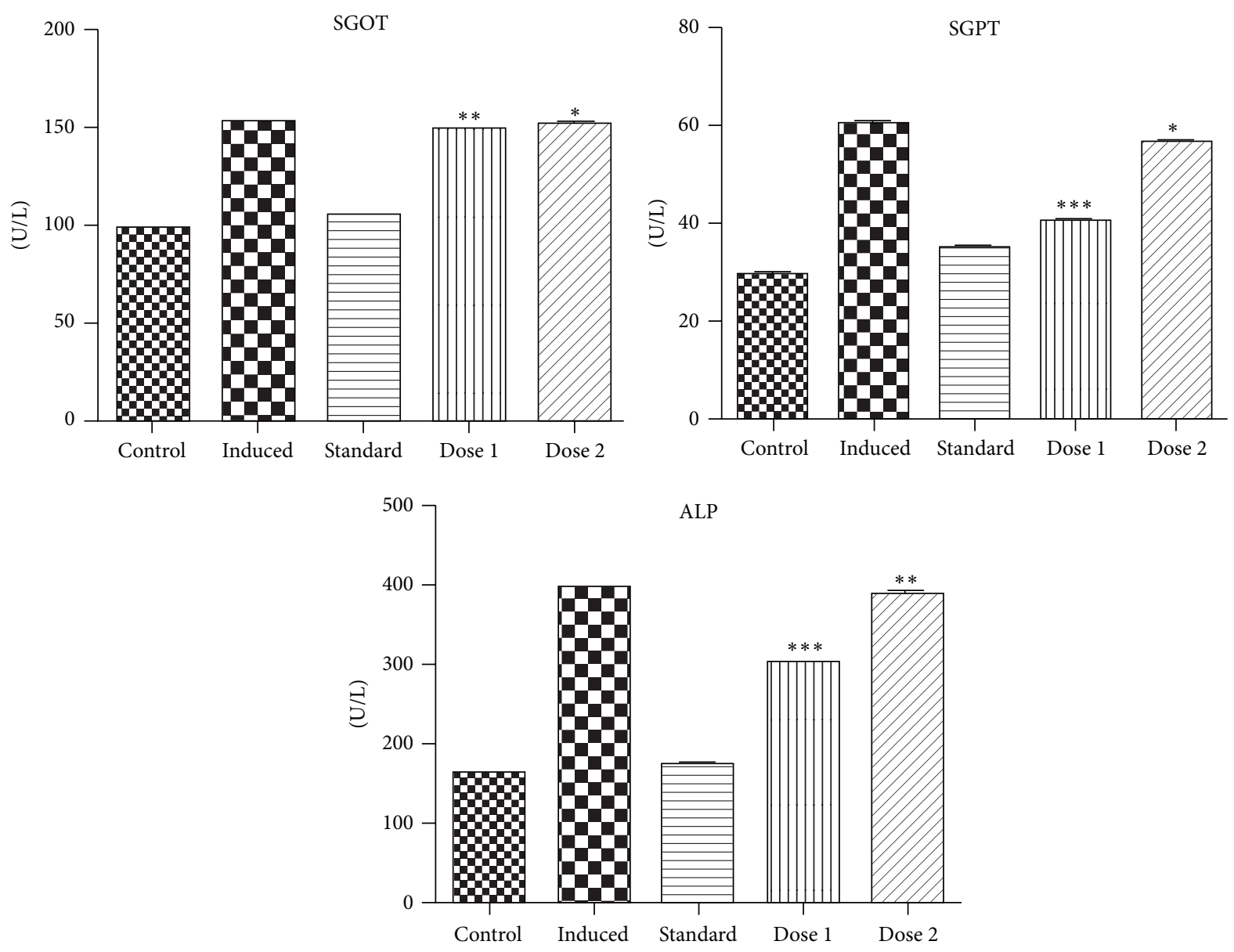

FIGURE 1: Bar diagram exhibits concentration of SGOT, SGPT, and ALP in rats belonging to five different groups.

\section{Results}

3.1. Indices of Hepatotoxicity. In the enzymatic assay, SGOT, SGPT, and ALP levels were analyzed in experimental groups. The level of SGOT was elevated in nanoparticles exposed group. However, the quinoline compounds IMDHPQ treatment showed significant reversal of the increased SGOT level. Meanwhile, in the case of SGPT, dosage one $(500 \mu \mathrm{L} / \mathrm{kg})$ decreased the increased level of SGPT and dosage two $(1000 \mu \mathrm{L} / \mathrm{kg})$ did not show much effect. Likewise, in the case of ALP too, dosage one showed better recovery than dosage two when compared to induced group (Figure 1).

3.2. Activity of Bilirubin and Protein. Bilirubin levels were estimated in the present study. It was elevated in nanoparticles induced group when compared to control group. However, with the administration of IMDHPQ, the levels were recovered back to near normal level in both doses. Likewise, total protein content was also elevated in nanoparticles induced group but, with the administration of quinoline compounds IMDHPQ, the protein content was lowered back to near normal level in dosage one compared to dosage two (Figure 2).
3.3. Effect of IMDHPQ on Antioxidants Enzymes. Enzymatic antioxidants levels were estimated. The level of GSH in InTiO NPs induced group showed significant reduction with that of control counterpart. Nevertheless, treated IMDHPQ revealed significant reversal from the decreased level to near normal level in dosage one but dosage two showed only moderate increase. Likewise, the catalase activity showed considerable increase in dosage one treated group when compared to InTiO NP induces group, whereas, in dosage two, the level was insignificant. GPx level was greatly decreased in induced group when compared to that of control group and it was reverted back significantly when treated with dosage one of IMDHPQ. Interestingly, dosage two did not show much effect in both catalase and GPx (Figure 3).

3.4. Effect of IMDHPQ on TBARS. The level of MDA in liver tissues of nanoparticle intoxicated rats was significantly elevated when compared to that of control animals. The administration of IMDHPQ reverted the MDA level convincingly in both doses (Figure 4).

3.5. Histopathology. Histopathology of liver sections was analyzed. InTiO NPs exposed liver sections exhibited normal 

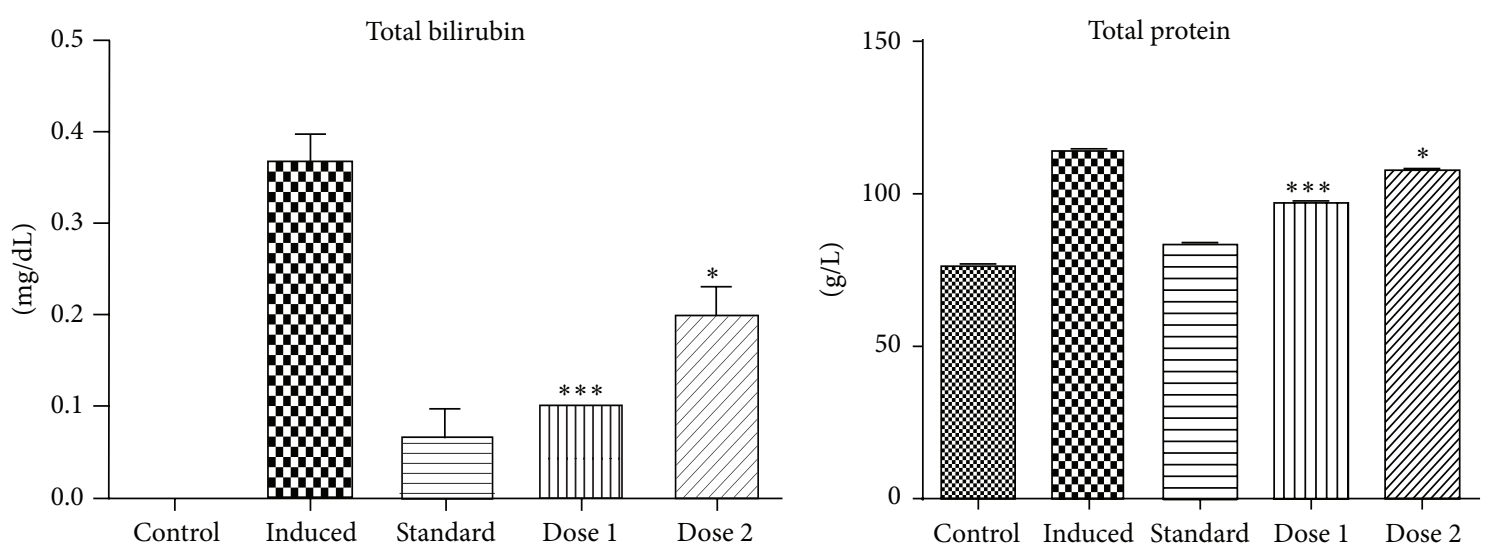

FIGURE 2: Bar diagram shows the level of total bilirubin and total protein in experimental rats.
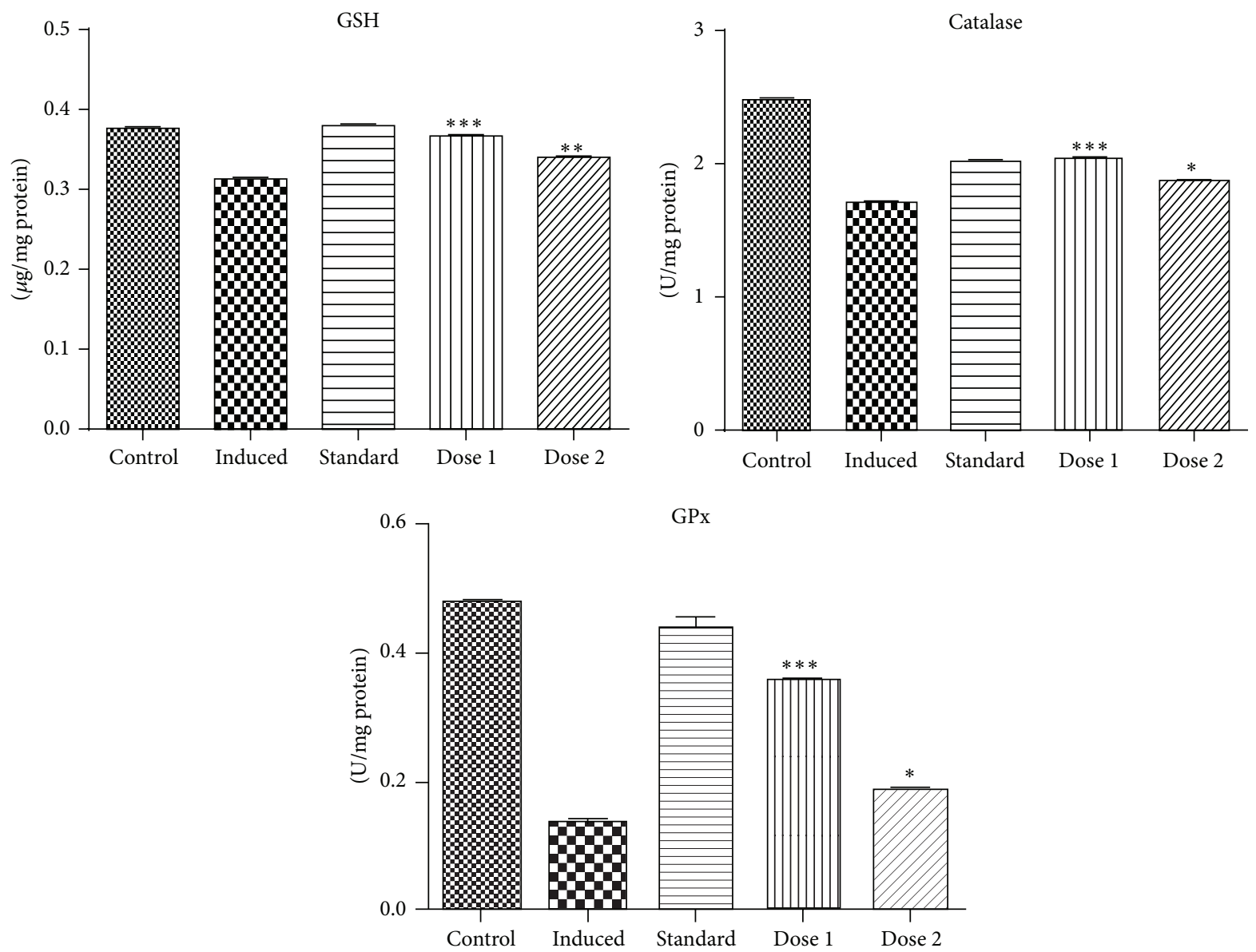

FIGURE 3: Bar diagram representing the average concentration of GSH, GPx, and catalase in rats belonging to five different groups.

lobular architecture but the portal tract and liver parenchyma showed few granulomas with mild inflammation. Sinusoids were mildly dilated. The damage caused due to NPs mediated oxidative stress was partially cured when the damaged group was treated with quinoline compound IMDHPQ as evinced by the presence no fibrosis, granuloma, inflammation, or fatty changes (Figure 5).

\section{Discussion}

In the present study, hepatotoxic effect of InTiO NP in albino rats was demonstrated. The International Program on Chemical Safety (1982) showed that most ingested titanium is excreted with urine and not absorbed by organisms. This implies that indium particles had a stronger toxicity to liver 


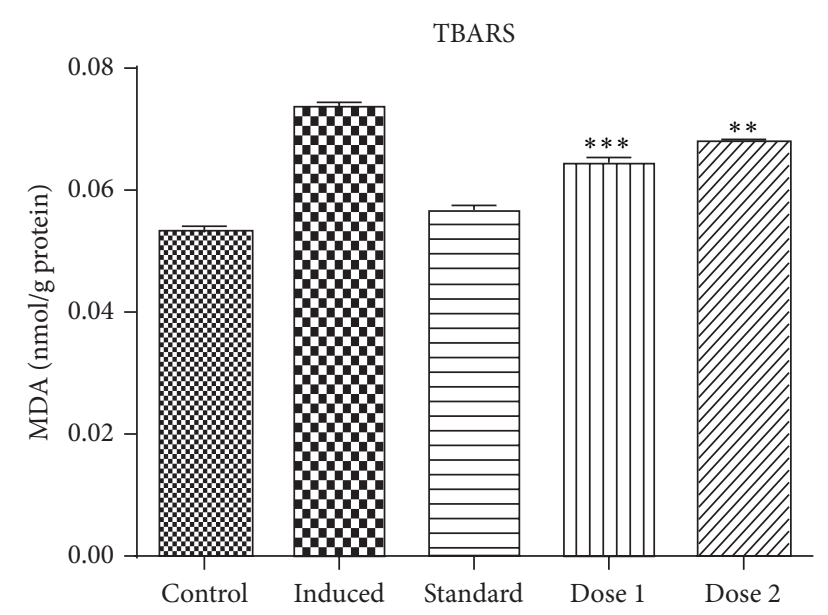

FIgURE 4: Bar diagram represents the level of MDA products in TBARS assay.

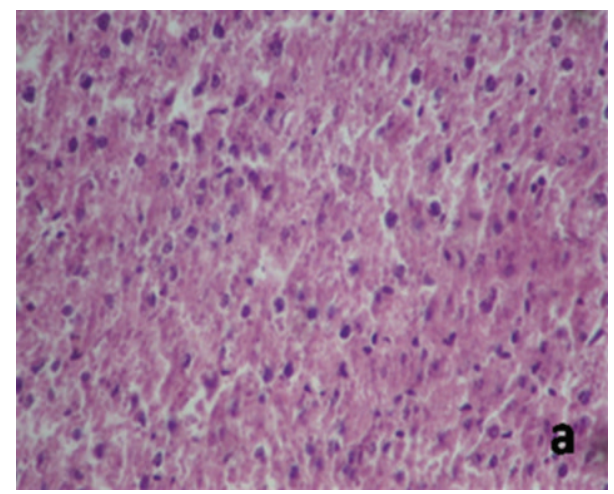

(a) InTiO NPs exposed liver shows mild degeneration

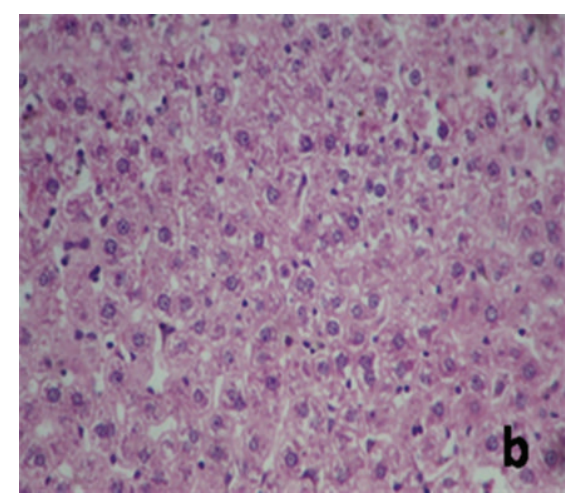

(b) Treated quinoline compound IMDHPQ shows regeneration of the damaged tissues

FIGURE 5: Histopathology of liver sections of experimental animals.

than to kidney. Also, the variation in liver-linked enzymatic activity could be associated with enzymatic variation that could be observed on the basis of age, sex, and other factors. Previous studies indicated that nano-sized particles could cross the small intestine and further distribute into blood, brain, lung, heart, kidney, spleen, liver, intestine, and stomach [17]. Therefore, it is possible that InTiO NP could accumulate in and transport to other tissues after oral administration. However, Chen et al. [17] reported that the different exposure routes, such as inhalation, dermal contact, and different metal salts administration, could cause different toxic effects.

Quinoline compounds are proved to be effective in treating various ailments. Generally, phenolic hydroxyl group and imine unit of the quinoline moiety are responsible for radical scavenging function. Several reports have ascribed it to the phenolic hydroxyl group which is preferred over "NH" group [18]. The antioxidant principles are involved in the organ protective activity. Hence, in the present study, the increased level of GSH and decreased lipid per oxidation can be attributed to the presence of antioxidant principle. The high dose in the range of $1000 \mu \mathrm{L}$ showed less significant action when compared to the first dose of $500 \mu \mathrm{L}$. The dose $(500 \mu \mathrm{L} / \mathrm{Kg})$ of quinoline administration proved to be protective to liver, whereas the $1000 \mu \mathrm{L}$ dose was insignificant. However, the treatment of IMDHPQ showed not much effect in the case of catalase and GPx.

Liver enzymes such as SGOT, SGPT, and ALP and bilirubin levels were estimated to assess the level of damage caused by InTiO NPs. The nanoparticle induced group showed rise in the level when compared to normal control rats. This is due to the fact that hepatic cells possess a variety of metabolic activities and contain several enzymes. SGPT and SGOT are found in higher concentration in cytoplasm and SGPT is found particularly in mitochondria. In liver injury, the transport function of hepatocytes is disturbed, resulting in the leakage of plasma membrane, thereby causing leakage of such enzymes leading to the increased serum levels [19]. In the present investigation, the elevated levels of SGOT, SGPT, and ALP and bilirubin in nanoparticle induced liver injury in serum are indicative of cellular leakage and loss of functional integrity of cell membrane in liver as attributed earlier [19].

SGPT is the best parameter compared to SGOT to justify the liver damage, since SGOT is also present in kidney and cardiac muscle. Treatment with the quinoline compound decreased the elevated levels of SGPT and SGOT which may be a consequence of the stabilization of plasma membrane as well as repair of hepatic tissue damage caused by the nanoparticles. This may be supported by a view of the healing of hepatic parenchyma and regeneration of hepatocytes. Serum ALP and bilirubin levels, on the other hand, are related to the function of hepatic cell. The compound showed dose dependent significant reduction in serum ALP and bilirubin, indicating an improvement in the secretary mechanism.

Lipid peroxidation is a destructive process in liver injury due to administration of hepatotoxicants. In the present study, the elevation in the level of TBARS in rat liver was observed. The increase in TBARS level suggests enhanced lipid peroxidation during tissue damage and failure of antioxidant defense mechanism to prevent formation of excessive free radicals $[20,21]$. However, quinoline compound has significantly inhibited the lipid peroxidation. It may be possible that mechanism of inhibition of lipid peroxidation may be due to its antioxidant effect and beneficial action 
against pathophysiological alterations caused by InTiO NPs mediated tissue damaging radicals.

Glutathione is one of the most abundant tripeptide antioxidants present in liver. In the present investigation, InTiO NPs have depleted the GSH, GPx, catalase, and total protein level with an association of increased lipid peroxidation, which leads to tissue injury and liver damage. Nevertheless, treatment with quinoline compound IMDHPQ reversed the changes occurring due to nanoparticle exposure. The coordinate action of antioxidant system is very critical for the detoxification of free radicals. CAT and GSHPx decompose $\mathrm{H}_{2} \mathrm{O}_{2}$ and protect the tissues from highly reactive hydroxyl radicals. In this study, quinoline compound IMDHPQ alleviated the InTiO NPs mediated oxidative stress with an increase in activities of antioxidant enzymes CAT and GSH-Px, in hepatic samples in dose dependent manner. The above results suggest that 2-imino-4-methyl1, 2-dihydropyrimido $[5,4 \mathrm{C}]$ quinoline-5(6H)-one possesses hepatoprotective property $[11,22]$.

Pathology of the organs due to nanomaterials toxicity was reported by many authors $[23,24]$ and primary organs like liver, kidney, lung, heart, and so forth were affected [23]. Our study shows that InTiO NP particles had stronger toxicity to liver than to kidney as this was further confirmed by the histopathological observations. Microscopic analysis of tissue sections of experimental animals revealed the damage caused by InTiO NPs. Portal tract and liver parenchyma showed few granulomas with mild inflammation. Sinusoids were mildly dilated. Generation of ROS may lead to malondialdehyde production and hepatocyte apoptosis. Swelling of liver cells exposed to nano-copper particles was reported [25]. Liver, kidneys, and spleen were gravely harmed by exposure to nano-copper particle [25]. Previous reports support the results of the present study. However, the damage caused due to NPs mediated oxidative stress was partially reverted when the damaged group was treated with quinoline compound IMDHPQ as evinced by the presence of no fibrosis in treated group. Beneficial effects of the quinoline compound were illustrated in previous study. It may be due to the presence of phenolic hydroxyl, imine unit, and the flavins like structure of the compound [11, 22].

\section{Conclusion}

In conclusion, altered biochemical, antioxidant, and histopathological profiles due to InTiO NPs toxicity were seen in the present study. Treatment with 2-imino-4-methyl-1, 2-dihydropyrimido $[5,4 \mathrm{C}]$ quinoline-5(6H)-one in nanoparticles induced group showed convincing hepatoprotective role in experimental rats. Further, detailed study of InTiO NPs induced oxidative stress mediated signaling mechanism and appropriate validation of administration of quinoline compounds for hepatotoxicity is needed.

\section{Disclosure}

Dinesh Bheeman and Sinjula Cheerothsahajan are co-first authors.

\section{Conflict of Interests}

The authors declare that there is no conflict of interests regarding the publication of this paper.

\section{Acknowledgments}

Sathish Sugumaran is thankful to the Jawaharlal Nehru Memorial Fund (JNMF), Doctoral Fellowship (SUA/797/2011-12), New Delhi. The authors are thankful to Ms. Punithavathi for her technical help.

\section{References}

[1] D. Y. Joh, J. Kinder, L. H. Herman et al., "Single-walled carbon nanotubes as excitonic optical wires," Nature Nanotechnology, vol. 6, no. 1, pp. 51-56, 2011.

[2] F. Tang, L. Li, and D. Chen, "Mesoporous silica nanoparticles: synthesis, biocompatibility and drug delivery," Advanced Materials, vol. 24, no. 12, pp. 1504-1534, 2012.

[3] A. M. Alkilany, J. Catherine, and C. J. Murphy, “Toxicity and cellular uptake of gold nanoparticles: what we have learned so far?" Journal of Nanoparticle Research, vol. 12, no. 7, pp. 23132333, 2010.

[4] C. Bruzeam, I. Ivan, B. Pacheco, and R. Kevin, "Nanomaterials and nanoparticles: sources and toxicity," Biointerphases, vol. 2, no. 4, pp. MR17-MR71, 2007.

[5] S. Arora, J. M. Rajwade, and K. M. Paknikar, "Nanotoxicology and in vitro studies: the need of the hour," Toxicology and Applied Pharmacology, vol. 258, no. 2, pp. 151-165, 2012.

[6] B. Trouiller, R. Reliene, A. Westbrook, P. Solaimani, and R. H. Schiestl, "Titanium dioxide nanoparticles induce DNA damage and genetic instability in vivo in mice," Cancer Research, vol. 69, no. 22, pp. 8784-8789, 2009.

[7] D. Lison, J. Laloy, I. Corazzari et al., "Sintered indium-tinoxide (ITO) particles: a new pneumotoxic entity," Toxicological Sciences, vol. 108, no. 2, pp. 472-481, 2009.

[8] R. Ahsan, K. M. Islam, A. Musaddik, and E. Haque, "Hepatoprotective activity of methanol extract of some medicinal plants against carbon tetrachloride induced hepatotoxicity in albino rats," Global Journal of Pharmacology, vol. 3, p. 116, 2009.

[9] S. K. Dubey and A. Batra, "Hepatoprotective activity from ethanol fraction of Thujaoccidentalis linn," Asian Journal of Research in Chemistry, vol. 1, no. 1, pp. 32-35, 2008.

[10] A. A. Patel and A. G. Mehta, "Synthesis of novel heterocyclic compounds and their biological evaluation," Der Pharma Chemica, vol. 2, no. 1, pp. 215-223, 2010.

[11] M. Sankaran, C. Kumarasamy, U. Chokkalingam, and P. S. Mohan, "Synthesis, antioxidant and toxicological study of novel pyrimido quinoline derivatives from 4-hydroxy-3-acyl quinolin-2-one," Bioorganic \& Medicinal Chemistry Letters, vol. 20, no. 23, pp. 7147-7151, 2010.

[12] D. Bheeman, S. Sugumaran, R. Mathan, S. Dakshanamoorthy, and C. S. Bellan, "Oxidative stress induced biochemical alterations due to novel InTiO nanoparticles exposure," Nanoscience and Nanotechnology Letters, vol. 6, no. 6, pp. 457-463, 2014.

[13] A. K. Sinha, "Colorimetric assay of catalase," Analytical Biochemistry, vol. 47, no. 2, pp. 389-394, 1972.

[14] M. S. Moron, J. W. de Pierre, and B. Mannervik, "Levels of glutathione, glutathione reductase and glutathione S-transferase 
activities in rat lung and liver," Biochimica et Biophysica Acta, vol. 582, no. 1, pp. 67-78, 1979.

[15] J. T. Rotruck, A. L. Pope, H. E. Ganther, A. B. Swanson, D. G. Hafeman, and W. G. Hoekstra, "Selenium: biochemical role as a component of glutathione peroxidase," Science, vol. 179, no. 4073, pp. 588-590, 1973.

[16] M. Burstein, H. R. Scholnick, and R. Morfin, "Rapid method for the isolation of lipoproteins from human serum by precipitation with polyanions," Journal of Lipid Research, vol. 11, no. 6, pp. 583-595, 1970.

[17] Z. Chen, H. Meng, G. Xing et al., "Acute toxicological effects of copper nanoparticles in vivo," Toxicology Letters, vol. 163, no. 2, pp. 109-120, 2006.

[18] K. I. P. Indira, "Free radical reactions of curcumin in membrane models," Free Radical Biology \& Medicine, vol. 23, no. 6, pp. 838843, 1997.

[19] S. Aneja, M. Vats, S. Aggarwal, and S. Sardana, "Phytochemistry and hepatoprotective activity of aqueous extract of Amaranthus tricolor Linn. roots," Journal of Ayurveda and Integrative Medicine, vol. 4, no. 4, pp. 211-215, 2013.

[20] H.-Y. Kim, J.-K. Kim, J.-H. Choi et al., "Hepatoprotective effect of pinoresinol on carbon tetrachloride-induced hepatic damage in mice," Journal of Pharmacological Sciences, vol. 112, no. 1, pp. 105-112, 2010.

[21] M. Daryoush, A. T. Bahram, D. Yousef, and N. Mehrdad, "Protective effect of turnip root (Brassica Rapa. L) ethanolic extract on early hepatic injury in alloxanized diabetic rats," Australian Journal of Basic and Applied Sciences, vol. 5, no. 7, pp. 748-756, 2011.

[22] L. Yan, P. Bjork, R. Butuc, J. Gawdzik, J. Earley, and G. Kim, "Beneficial effects of quinoline-3-carboxamide (ABR-215757) on atherosclerotic plaque morphology in S100A12 transgenic ApoE null mice," Atherosclerosis, vol. 228, no. 1, pp. 69-79, 2013.

[23] Y. Zheng, R. Li, and Y. Wang, "In vitro and in vivo biocompability of ZnO nanoparticles," International Journal of Modern Physics B, vol. 23, pp. 1566-1571, 2009.

[24] B. Wang, W. Feng, M. Wang et al., "Acute toxicological impact of nano- and submicro-scaled zinc oxide powder on healthy adult mice," Journal of Nanoparticle Research, vol. 10, no. 2, pp. 263276, 2008.

[25] J. Li, E. S. Quabius, S. E. Wendelaar Bonga, G. Flik, and R. A. C. Lock, "Effects of water-borne copper on branchial chloride cells and $\mathrm{Na}^{+} / \mathrm{K}^{+}$-ATPase activities in Mozambique tilapia (Oreochromis mossambicus)," Aquatic Toxicology, vol. 43, no. 1, pp. 1-11, 1998. 

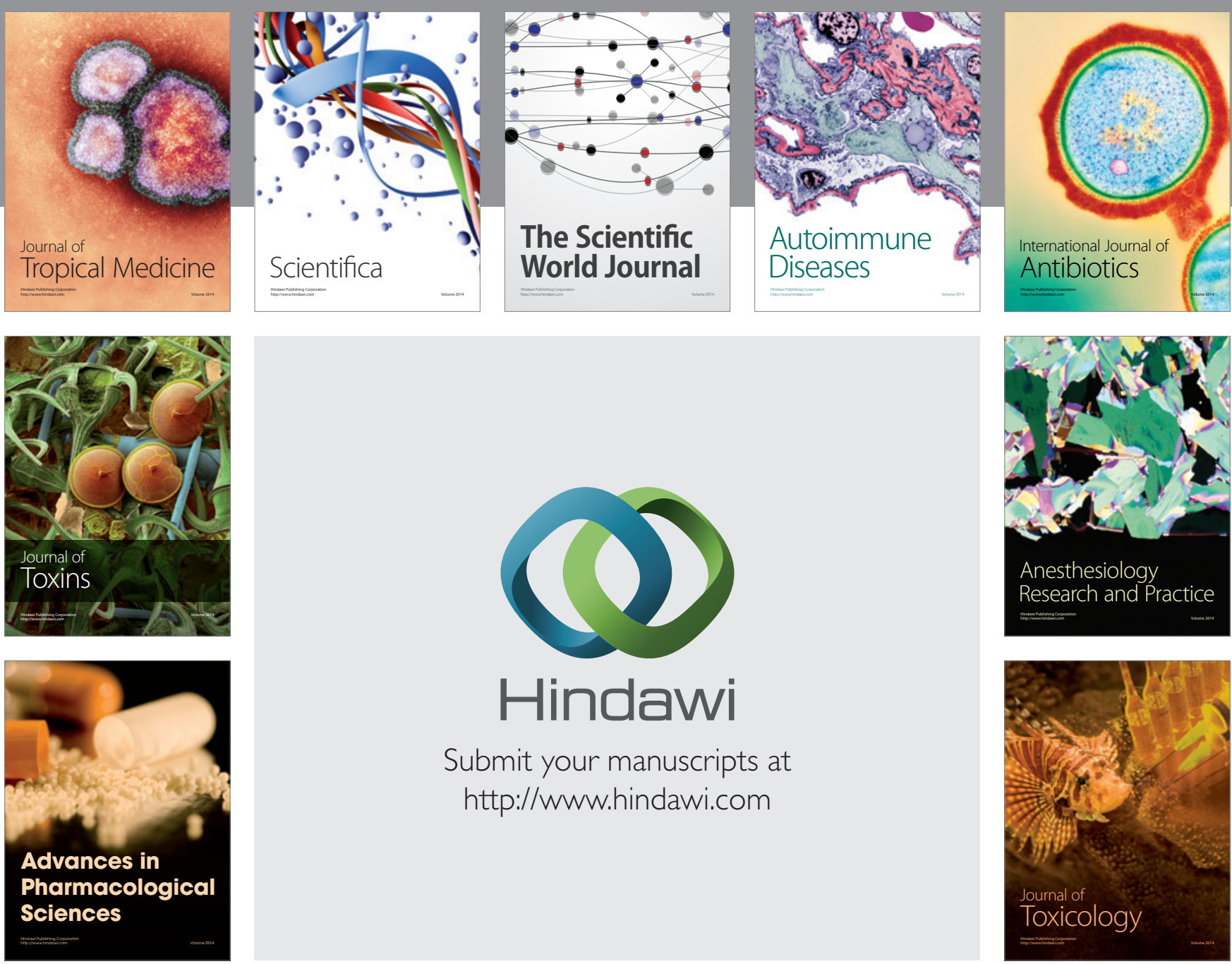

\section{Hindawi}

Submit your manuscripts at

http://www.hindawi.com
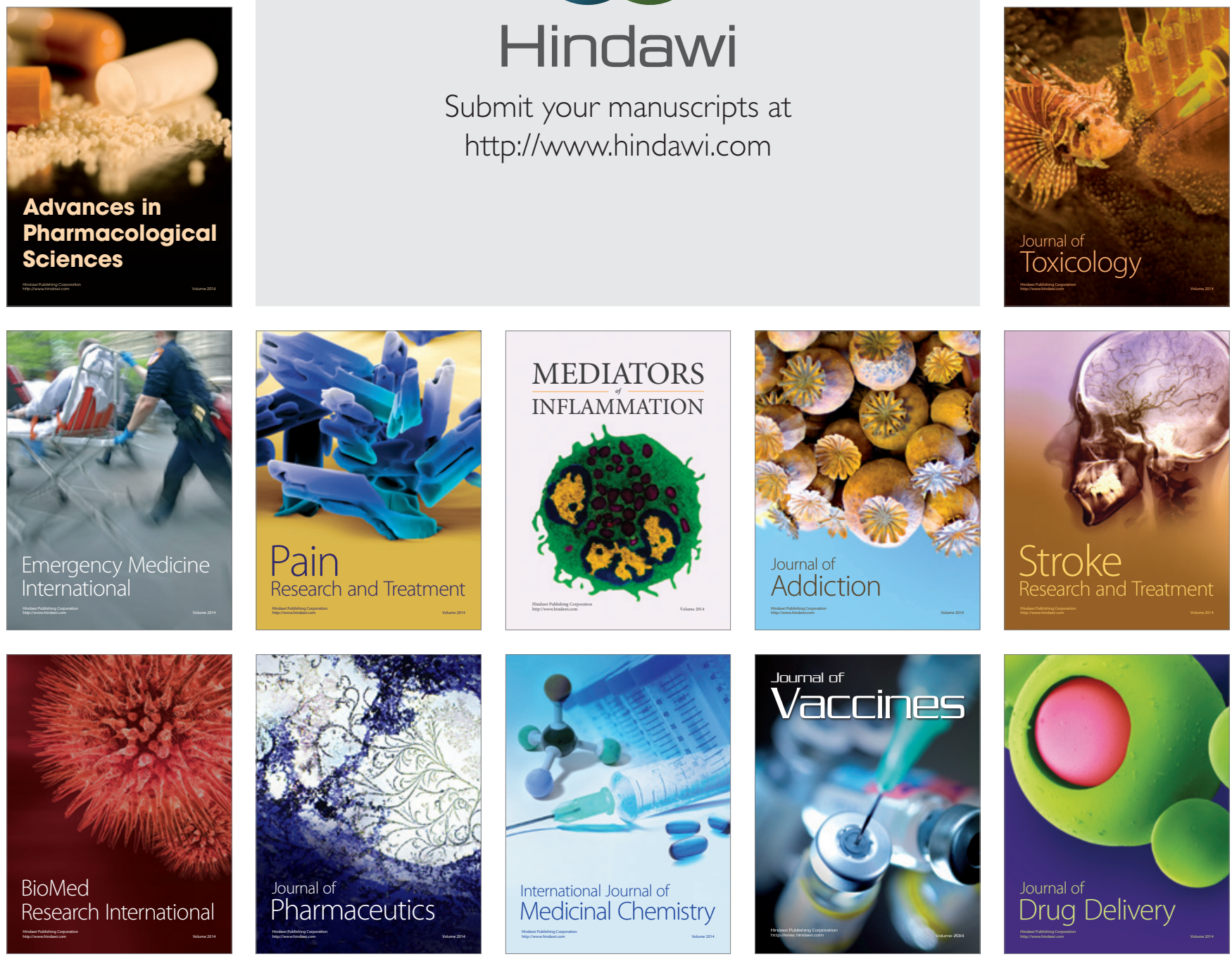